ORIGINAL ARTICLE

\title{
Inflammatory cells contribute to the generation of an angiogenic phenotype in pancreatic ductal adenocarcinoma
}

\section{Esposito, M Menicagli, N Funel, F Bergmann, U Boggi, F Mosca, G Bevilacqua, D Campani}

J Clin Pathol 2004;57:630-636. doi: 10.1136/icp.2003.014498

See end of article for authors' affiliations

Correspondence to: Dr I Esposito, Institute of Pathology, University of Heidelberg, Im

Nevenheimer Feld 220 69120 Heidelberg,

Germany; irene_esposito@ med.uni-heidelberg.de

Accepted for publication 22 December 2003

\begin{abstract}
Background: Inflammatory cells contribute to the growth and spread of human malignancies by producing molecules that enhance tumour invasiveness.

Aims: To characterise the inflammatory infiltrate in pancreatic ductal adenocarcinoma and to analyse its contribution to angiogenesis and its prognostic relevance.

Methods: Immunohistochemistry was used to identify inflammatory cells and evaluate the expression of proangiogenic and prolymphangiogenic molecules (vascular endothelial growth factor A (VEGF-A), VEGF-C, and basic fibroblast growth factor (bFGF)) by inflammatory and cancer cells in 137 pancreatic cancers. Intratumorous microvessel density (IMD) was assessed using CD34 as an endothelial cell marker. Results: There were significantly more mast cells and macrophages in pancreatic cancers than in normal pancreas and the number of mast cells directly correlated with the presence of lymph node metastases. However, there was no relation between numbers of infiltrating inflammatory cells and the presence of chronic pancreatitis (CP)-like changes in the parenchyma surrounding the tumour. Double immunostaining revealed that both pancreatic mast cells and macrophages express VEGF-A, VEGF-C, and bFGF. These factors were also expressed in the tumour cells in many cases. The numbers of VEGF-A expressing tumour cells and bFGF expressing tumour and inflammatory cells significantly correlated with IMD. Moreover, tumours with higher IMD had higher numbers of infiltrating mast cells and macrophages.

Conclusions: Mononuclear inflammatory cells of the non-specific immune response are recruited to pancreatic cancer tissues independent of the presence of CP-like changes, may influence the metastatic capacity of the cancer cells, and may contribute to the development of tumours with high angiogenic activity.
\end{abstract}

$P$ ancreatic adenocarcinoma is characterised by a dense, desmoplastic stroma rich in collagen fibres, extracellular matrix proteins, fibroblasts, and inflammatory cells. Recent studies have analysed the relation between tumour cells and the surrounding stroma in pancreatic cancer, and have demonstrated the existence of spatially distinct compartments of gene expression that lead to the establishment of a sort of crosstalk between the connective tissue cells and the cancer cells. ${ }^{23}$ According to these studies, molecules related to the immune response, such as immunoglobulin light and heavy chains, are selectively overexpressed in the tumour stroma. ${ }^{2}$ This fact supports the existence of an antitumour immune response exerted by the inflammatory cells that populate the peritumorous connective tissue. However, inflammatory cells may take part in the process of tumour invasion: tumour cells produce cytokines and growth factors that promote the growth of fibroblasts and may have chemotactic effects on inflammatory cells, which in turn synthesise cytokines, growth factors, and proangiogenic factors that contribute to cancer progression. ${ }^{45}$ In addition, it is well known that the infiltration of inflammatory cells into a defined site of the organism can precede the development of a neoplasm and that a pre-existing inflammatory, often infective, condition can be involved in the pathogenesis of many human malignancies. Examples include gastric carcinomas that arise in a Helicobacter pylori induced gastritis environment or hepatitis B virus/hepatitis C virus related hepatocellular carcinoma. In some settings, a chronic, noninfective inflammatory condition is responsible for the promotion phase of carcinogenesis, as in the case of smoking related bronchial cancer. ${ }^{6}$ Pancreatic cancer may also develop in the context of a chronic inflammatory condition. Chronic pancreatitis is recognised as a definite risk factor for the development of pancreatic cancer, and many of the growth promoting factors that are involved in tissue remodelling and regeneration in chronic pancreatitis are frequently overexpressed in pancreatic cancer. ${ }^{78}$ However, the mechanisms that connect inflammatory cells to the development of pancreatic cancer are not clear. The involvement of macrophages in the proliferation and invasive capacity of pancreatic tumour cells and in the process of angiogenesis through the production of the macrophage proinflammatory chemokine$3 \alpha$, has been postulated. ${ }^{9}$ The contribution of macrophages to the growth of tumour cells has been described in other human malignancies. For example, in breast cancer the expression of colony stimulating factor 1 (CSF-1) correlates with an intense macrophage infiltration and with poor prognosis; moreover, transgenic mice bearing a recessive null mutation in the CSF-1 gene develop mammary tumours with little inflammatory infiltration and low metastatic potential. ${ }^{10}$ Mast cells frequently accumulate in human tumours and tend to concentrate at tumour borders, as first described by Westphal in $1891 .{ }^{11}$ A relation between the number of mast cells and the invasive ability of the tumour cells has been described in melanomas and lung and oesophageal carcinomas, and has been mainly related to the production of proangiogenic factors by mast cells. ${ }^{12-14}$ However, in a previous series of 26 cases of pancreatic adenocarcinoma,

Abbreviations: bFGF, basic fibroblast growth factor; CSF, colony stimulating factor $1 ; I M D$, intratumorous microvessel density; VEGF, vascular endothelial growth factor 
no correlation between mast cell infiltrates and pathological parameters of local and distant invasion was found. ${ }^{15}$

\section{"The mechanisms that connect inflammatory cells to the development of pancreatic cancer are not clear"}

The purpose of our study was to characterise the mononuclear inflammatory cells in pancreatic ductal adenocarcinoma specimens and to assess their role in the production of proangiogenic growth factors that may influence the intratumorous microvessel density (IMD) and contribute to the local and distant spread of the cancer cells.

\section{METHODS \\ Patients}

Pancreatic adenocarcinoma tissue samples were obtained from patients $(\mathrm{n}=137 ; 66$ men, 71 women; median age, 68 years; range, 37-88) undergoing surgery for pancreatic cancer between January 1996 and October 2002. In 70 patients $(51 \%)$ a pylorus preserving pancreaticoduodenectomy was performed; 30 patients $(22 \%)$ underwent a pancreatic left resection, 26 patients (19\%) had a classic Whipple resection, and $11(8 \%)$ had a total pancreatectomy. In one case, the tumour was a pancreatic body recurrence in a patient operated on four years before for ductal adenocarcinoma of the pancreatic head. One patient received preoperative chemotherapy. Follow up data were available for 79 patients. Of these, 14 patients ( $18 \%$ ) were disease free and alive with a follow up of 14-89 months (median, 27 months). Sixty five patients $(82 \%)$ died as a result of disease recurrence; survival time was calculated from the date of surgery until the date of death.

Ten samples from non-neoplastic pancreatic tissues were obtained through an organ donor programme.

\section{Pathological analysis}

Tissue samples were received fresh from the operating theatre immediately after removal, fixed in $10 \%$ buffered formalin, and embedded in paraffin wax for subsequent routine histopathological examination. Morphological classification and grading were conducted according to the World Health Organisation system ${ }^{16}$; staging was assessed according to the TNM classification of the International Union Against Cancer $2002 .{ }^{17}$

\section{Immunohistochemistry}

Consecutive paraffin wax embedded tissue sections $2-4 \mu \mathrm{m}$ thick were subjected to immunostaining with the streptavidin-peroxidase technique, using the automated NexES IHC staining system (Ventana, Tucson, Arizona, USA).

The following primary antibodies directed against human proteins were used: mouse anti-basic fibroblast growth factor (anti-bFGF; clone bFM-2; 1/50 dilution; Upstate Biotechnology, Lake Placid, New York, USA), rabbit anti-vascular endothelial growth factor A (anti-VEGF-A; 1/50 dilution; Santa Cruz Biotechnology Inc, Santa Cruz, California, USA), goat anti-VEGF-C (Santa Cruz Biotechnology; 1/50 dilution), mouse anti-CD34 (clone QBEnd/10; prediluted; Ventana Medical Systems), mouse antitryptase (clone G3; 1/1500 dilution; Chemicon International, Temecula, California, USA), and mouse anti-CD68 (clone KPl; 1/100 dilution; Dako, Carpenteria, California, USA). Pressure cooker heating (in citrate buffer, $\mathrm{pH}$ 7.3) was used as pretreatment for antigen retrieval for bFGF staining, microwave heating (in citrate buffer, $\mathrm{pH}$ 7.3) for VEGF-A, and protease digestion (protease 2; Ventana Medical Systems) for VEGF-C staining.
To ensure antibody specificity, consecutive sections were incubated with isotype matched control immunoglobulins and in the absence of primary antibody. In these cases, no specific immunostaining was detected.

\section{Double immunohistochemistry}

After an initial staining procedure, performed with the streptavidin-alkaline phosphatase technique (CD68 and tryptase), slides were immediately submitted to a second staining with the streptavidin-peroxidase technique (bFGF, VEGF-A, and VEGF-C), as described above, followed by haematoxylin counterstaining.

\section{Evaluation of the immunohistochemical results}

The immunohistochemical expression of VEGF-A, VEGF-C, and bFGF in the cancer cells was assessed using a semiquantitative method to evaluate both the staining intensity $(0$, negative; 1 , weak; 2 , moderate; 3 , strong) and the percentage of stained cells $(0,0 \%$ positive cells; $1,1-25 \%$ positive cells; $2,26-50 \%$ positive cells; $3,>50 \%$ positive cells). The sum of the two parameters reached a maximum score of 6. A score $>3$ was considered to be a positive immunohistochemical result.

To assess the number of mast cells and macrophages and the IMD each section was scanned at $\times 40$ magnification to identify the five areas (hot spots) with the highest number of mast cells (tryptase staining), macrophages (CD68 staining), and neo-vessels (CD34 staining). Perivascular areas, where inflammatory cells accumulate, were excluded. Then, each area was examined at $\times 200$ magnification $\left(0.949 \mathrm{~mm}^{2} /\right.$ field $)$ and the stained cells/vessels were counted. The mean value of the five areas was taken as representative of the whole section. The same procedure was applied to count the number of VEGF-A positive, VEGF-C positive, and bFGF positive inflammatory cells.

Assessment of the immunohistochemical staining was performed independently by two authors (IE and MM). Contrasting results were discussed until an agreement was reached.

\section{Statistical analysis}

The data are expressed as mean (SD). The Student's $t$ test was used for comparison of means, and categorical data comparison was carried out with the $\chi^{2}$ test. The Spearman rank correlation coefficient was used to determine correlations; one way analysis of variance was conducted with the ANOVA test. Survival analysis was performed with the Kaplan-Meier method and statistical comparisons were made with the log rank test. Significance was defined as $\mathrm{p}<0.05$.

\section{RESULTS}

\section{Pathological analysis}

All the tumours analysed in this series were ductal adenocarcinomas. Table 1 shows their distribution according to the TNM classification, their histopathological grading distribution, and their demographic characteristics.

In 74 patients (54\%), chronic pancreatitis-like alterations were present in the non-tumorous parenchyma; in 16 cases (11.6\%) an increase in the amount of periductal connective tissue (periductal fibrosis) was seen. The 10 non-neoplastic pancreas samples showed no signs of inflammation/fibrosis and were therefore considered as normal controls.

\section{Expression of VEGF-A, VEGF-C, and bFGF in pancreatic cancer specimens}

VEGF-A immunoreactivity was present as a diffuse staining in the cytoplasm of the cancer cells in 126 of 137 (92\%) cases. The percentage of positive cells ranged from $50 \%$ to $100 \%$ of 


\begin{tabular}{|ll|}
\hline $\begin{array}{l}\text { Table } 1 \\
\text { patients }\end{array}$ & Clinical and pathological characteristics of the \\
\hline Parameter & No. of cases (\%) \\
\hline Sex & \\
Male & $66(48)$ \\
Female & $71(52)$ \\
T & $2(2)$ \\
T1 & $2(2)$ \\
T2 & $107(78)$ \\
T3 & $26(19)$ \\
T4 & $41(30)$ \\
N & $96(70)$ \\
No & $1(1)$ \\
N1 & $2(2)$ \\
Tumour stage & $31(23)$ \\
IA & $69(50)$ \\
IB & $17(12)$ \\
IIA & $17(12)$ \\
IIB & \\
III & $17(12)$ \\
IV & $93(68)$ \\
Tumour grade & $27(20)$ \\
G1 & \\
G2 & $72(53)$ \\
G3 & $65(47)$ \\
Perineural infiltration & \\
Yes & \\
No & \\
\hline & \\
& \\
& \\
&
\end{tabular}

the cancer cells present in the tumour specimens and the intensity of the staining was moderate to intense. In all cases, the mononuclear inflammatory cells that were present in the tumour stroma also exhibited VEGF-A immunoreactivity (fig 1A). The numbers of VEGF-A positive inflammatory cells ranged from six to 278 , with a mean (SD) value of 73.8 (49.3), and there were significantly more in cases with VEGF-A positive tumour cells than in those with VEGF-A negative tumour cells $(77.3 \vee 33.6 ; \mathrm{p}=0.004)$.
VEGF-C staining was weak to intense in the cytoplasm of $40-100 \%$ of the tumour cells in 71 cases $(52 \%$ ) (fig 1B). Mononuclear inflammatory cells expressing VEGF-C were present in all the specimens (fig 1D), with numbers ranging from four to 154 (mean, 36.5; SD, 20.8), and no significant differences between cases with VEGF-C positive or negative tumour cells.

bFGF was expressed in the cytoplasm of the cancer cells in 107 cases $(78 \%$ ) (fig 1 C); $60-90 \%$ of the cancer cells exhibited weak to intense positivity. A mean number of 32.1 mononuclear inflammatory cells in each specimen (SD, 21.2; range, 4-130) showed bFGF immunoreactivity. The mean value of bFGF positive inflammatory cells was higher in cases with bFGF positive tumour cells than in those with bFGF negative tumour cells (35.07 $v 21.8 ; \mathrm{p}=0.002)$.

\section{Correlation of VEGF-A, VEGF-C, and bFGF expression with clinicopathological parameters}

No significant correlation was found between tumour cell VEGF-A, VEGF-C, and bFGF expression and the patients' sex, age, tumour size, TNM status, or grade of differentiation. VEGF-A positive tumours had wider local extension, expressed by the $\mathrm{T}$ status $(\mathrm{p}=0.053)$. The number of VEGF-A positive inflammatory cells correlated with the presence of lymph node metastases $(p=0.009)$; the number of VEGF-C positive inflammatory cells showed a tendency towards a correlation with the $\mathrm{N}$ status $(\mathrm{p}=0.06)$. No parameter correlated with perineural infiltration.

\section{Expression of VEGF-A, VEGF-C, and bFGF in the normal pancreas}

VEGF-A, VEGF-C, and bFGF were focally expressed in the acinar cells; moderate and focal expression of VEGF-A and bFGF was also seen in the epithelial cells of the normal ducts. Endocrine cells displayed a moderate to intense positivity for the three factors. In addition, occasional inflammatory cells seen in the connective tissue septa between the acini and around the ducts stained positively for VEGF-A, VEGF-C, and bFGF.
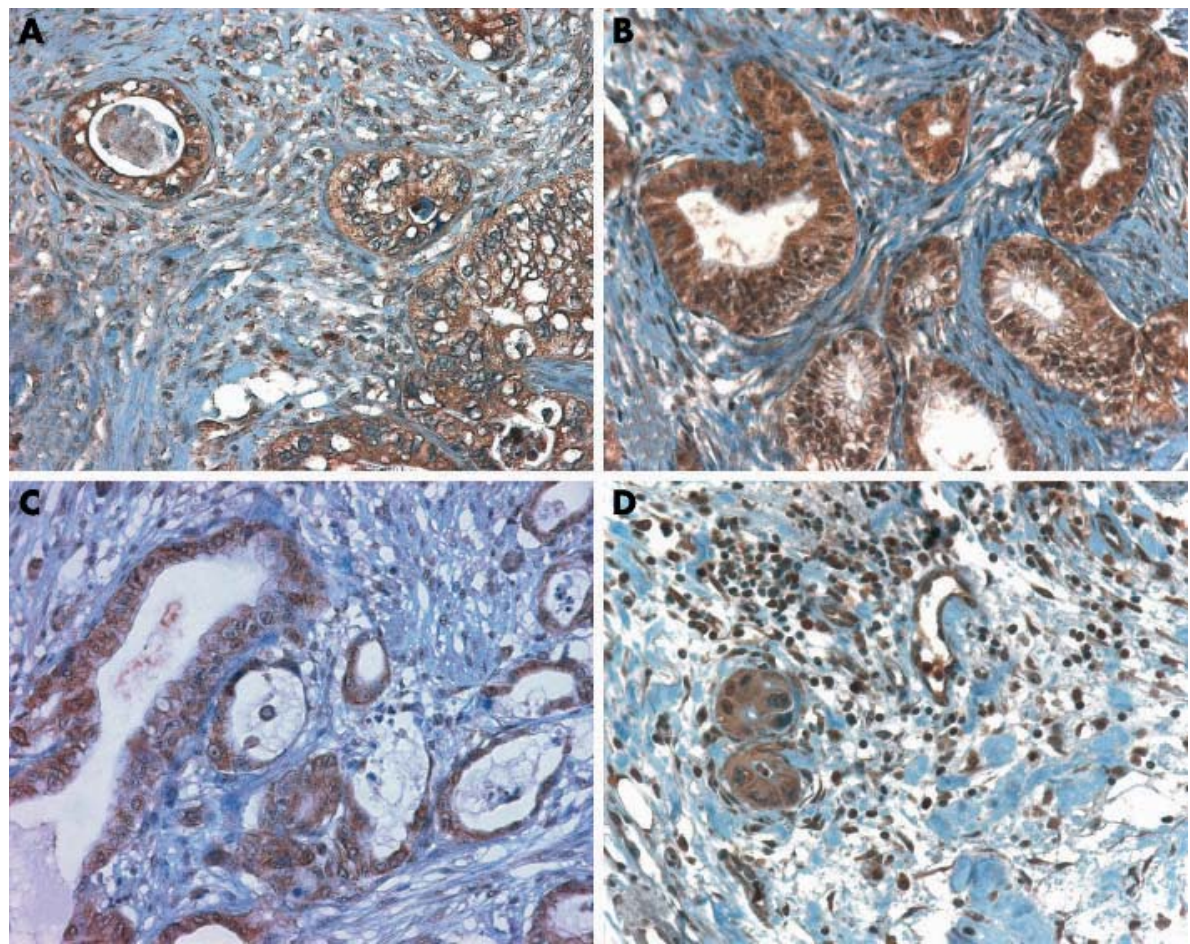

Figure 1 Expression of proangiogenic growth factors in pancreatic ductal adenocarcinoma. Strong immunoreactivity for (A) vascular endothelial growth factor A (VEGF-A), (B) VEGF-C, and (C) basic fibroblast growth factor in the cytoplasm of the tumour cells; positive inflammatory cells are visible in the stroma around the tumour cells and (D) at the tumour periphery. 


\section{Characterisation of the inflammatory infiltrate in pancreatic adenocarcinoma and comparison with the normal pancreas}

A morphological analysis of the tumour specimens, together with the immunohistochemical analysis, allowed the characterisation of the inflammatory cell infiltrate in pancreatic adenocarcinoma. Sparse mononuclear cells were present around the neoplastic glands, with a tendency to cluster at the peripheral, invasive edge of the tumour. With the use of specific monoclonal antibodies, most of these cells were identified as mast cells (tryptase positive) and macrophages (CD68 positive) (fig 2A, B). The mean number of mast cells was 61.3 (SD, 38.6; range, 7-235), whereas the mean number of macrophages was 61.4 (SD, 57.05; range, 1-363). Lymphocytes were easily identified on a morphological basis; they were present as part of the inflammatory infiltrate around the tumour glands or formed lymphoid follicles, mainly at the periphery of the tumour. Plasma cells were also occasionally present.

Mast cells and macrophages were more numerous in pancreatic cancer than in the normal pancreas. Here, the mean (SD) number of mast cells was 28.2 (15.4) $(p=0.002)$ and that of macrophages was 6.9 (9.3) $(p<0.0001)$. However, the accumulation of inflammatory cells in the tumour tissues was not influenced by the presence of chronic pancreatitis-like features in the surrounding parenchyma. When correlated with the clinical and pathological parameters, the number of mast cells showed an association with the presence of lymph node metastases $(\mathrm{p}=0.01)$.

By means of double immunohistochemistry, most of the VEGF-A, VEGF-C, and bFGF expressing inflammatory cells were identified as mast cells and macrophages (fig 2C, D).

\section{Neoangiogenesis in pancreatic adenocarcinoma}

A monoclonal antibody raised against the endothelial marker CD34 was used to detect the presence of newly formed vessels in the cancer specimens. The mean number of microvessels was 101.06 (SD, 70.2; range, 19-391). The expression of VEGF-A by the cancer cells was directly correlated with the IMD $(r=0.336 ; \mathrm{p}=0.0001)$; a direct correlation was also found between the expression of bFGF in the cancer cells and the IMD $(r=0.177 ; \mathrm{p}=0.04)$, and between the expression of bFGF in the inflammatory cells and the IMD $(r=0.217 ; \mathrm{p}=0.01)$. No significant correlation was found between the expression of VEGF-A in the inflammatory cells and the IMD. Neither the expression of VEGF-C in the cancer cells, nor the expression of VEGF-C in the inflammatory cells correlated with the IMD.

Interestingly, the numbers of infiltrating mast cells and macrophages showed a direct correlation with the IMD (mast cells and IMD: $r=0.256, p=0.003$; macrophages and IMD: $r=0.320, \mathrm{p}=0.0001$ ).

\section{Survival analysis}

The only parameter that significantly affected the patients' survival was the presence of distant metastases $(p=0.04)$. Neither the IMD nor the number of infiltrating inflammatory cells showed a significant impact on the patients' prognosis. However, when patients with low numbers of infiltrating mast cells $(\leqslant 25$ centile) were compared with those with high numbers of infiltrating mast cells ( $\geqslant 75$ centile), a tendency towards a longer survival of the first group (mean survival time, $19 v 16$ months) was seen. Moreover, patients with low numbers of infiltrating mast cells/macrophages and low IMD had a slightly better prognosis than those with high numbers of infiltrating mast cells/macrophages and high IMD (mean survival time $24 v 18$ months and $24 v 16$ months, respectively). However, these differences were not significant (fig 3).

\section{DISCUSSION}

The role of inflammatory cells in human cancer is controversial. The accumulation of inflammatory cells at tumour sites has been considered a mechanism of host defence. The growth of a neoplasm induces a specific, lymphocyte mediated immune response as a result of the expression of new antigens by the cancer cells and a non-specific inflammatory reaction, which follows tissue destruction and remodelling. Although the presence of infiltrating
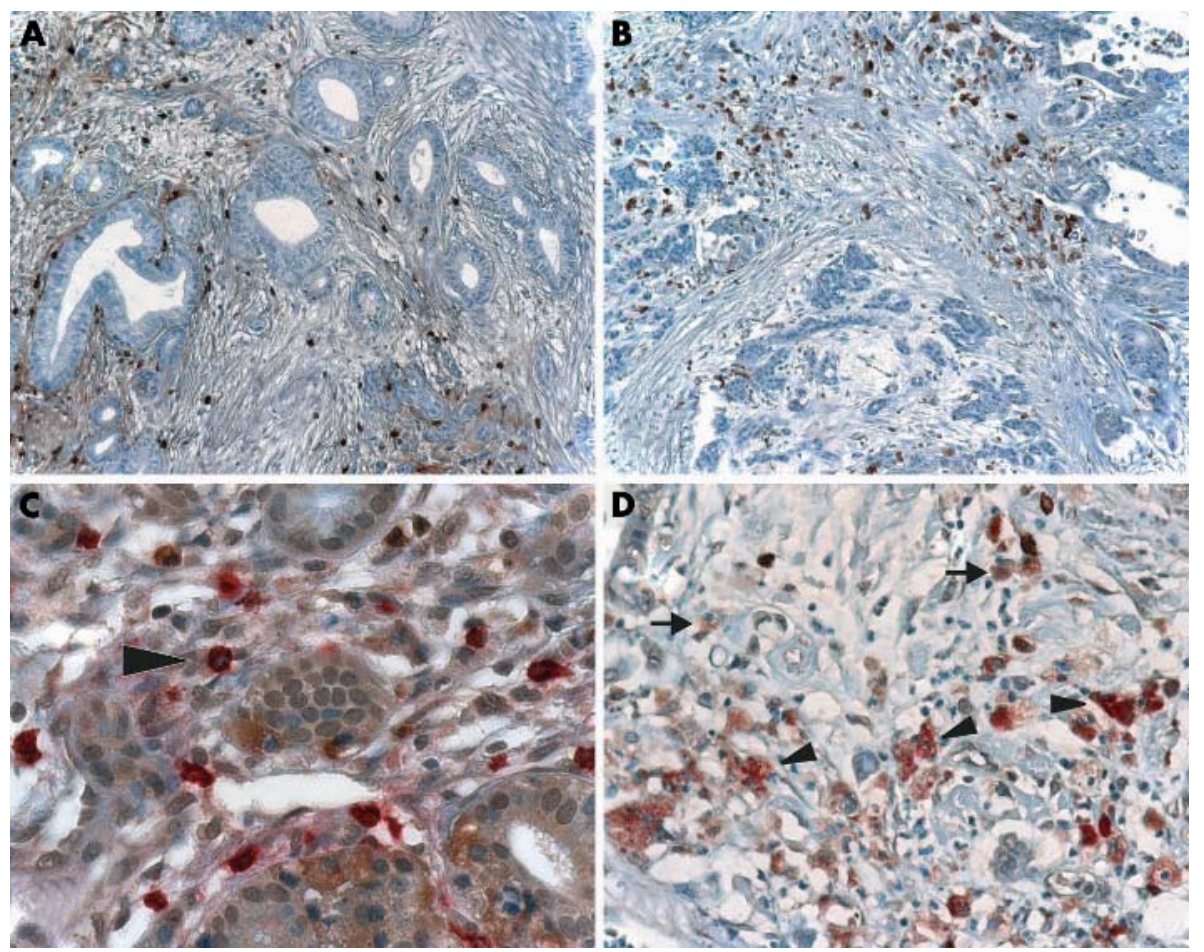

Figure 2 Characterisation of the inflammatory infiltrate in pancreatic ductal adenocarcinoma. (A) Tryptase positive mast cells and (B) CD68 positive macrophages are the most common inflammatory cells in the stroma of pancreatic ductal adenocarcinoma. Double immunohistochemistry shows that (C) mast cells and (D) macrophages (red staining) are a source of vascular endothelial growth factor C (brown staining) in pancreatic ductal adenocarcinoma. The arrowheads point to double positive inflammatory cells, the arrows to single (brown) stained inflammatory cells. 


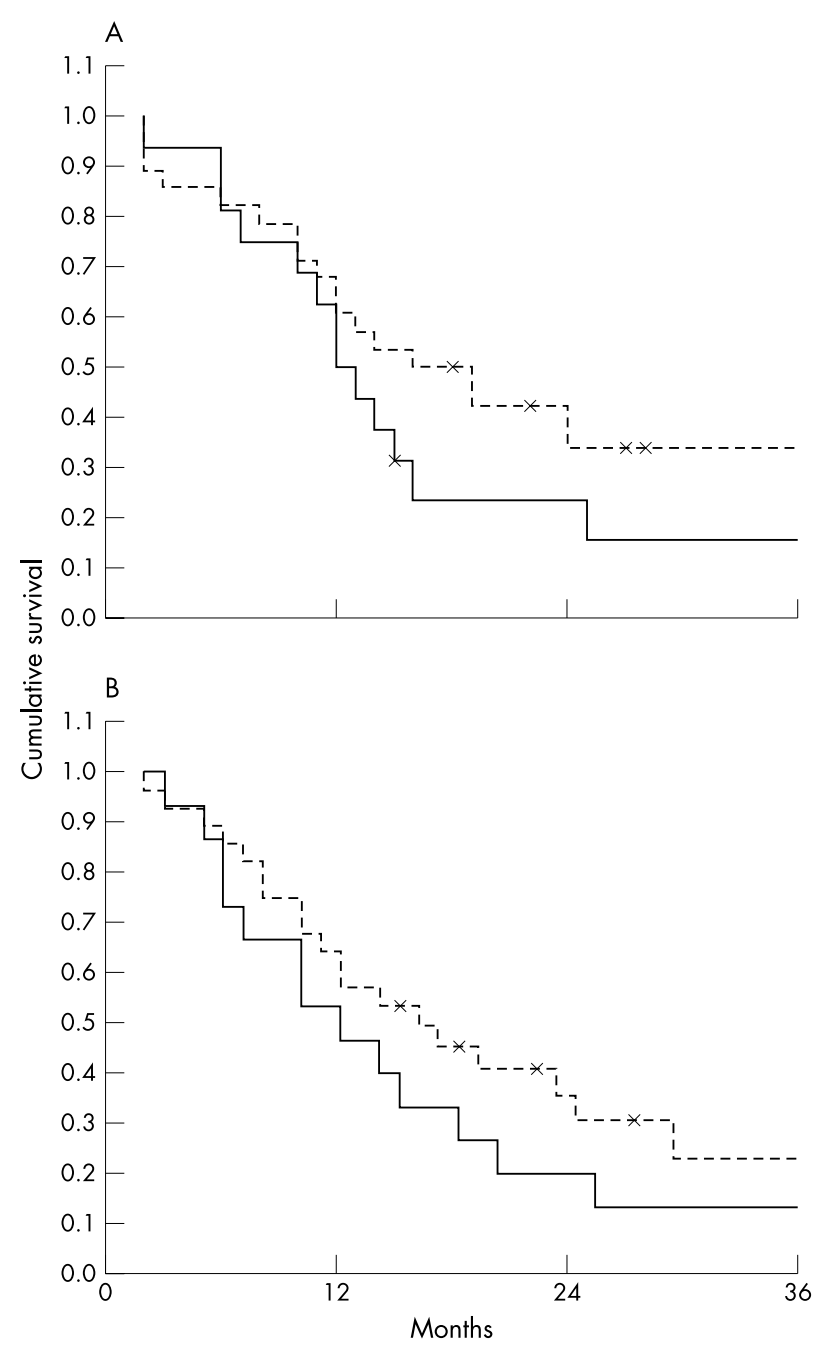

Figure 3 Survival analysis. (A) The survival of patients whose tumours exhibited a high number of infiltrating mast cells and a high ntratumorous microvessel density (IMD; solid line) was shorter than that of patients whose tumours exhibited a low number of infiltrating mast cells and a low IMD (broken line). However, the difference was not significant. (B) The same tendency was seen when the number of infiltrating macrophages was combined with the IMD (solid line, high number of macrophages and high IMD; broken line, low number of macrophages and low IMD). Crosses indicate censored cases.

inflammatory cells in a tumour mass represents the basis of the "immunosurveillance" against tumour growth, ${ }^{18}{ }^{19}$ many recent studies indicate that the inflammatory component of a developing neoplasm can provide useful means for cancer growth and spreading, ${ }^{20}{ }^{21}$ mostly by potentiating extracellular matrix remodelling and angiogenesis.

Our study has characterised the inflammatory cell infiltrate in pancreatic ductal adenocarcinoma, showing that mast cells and macrophages are the most common cell types of the nonspecific immune response. Moreover, they express proangiogenic growth factors-namely, VEGF-A, and, to a lesser extent, bFGF, and VEGF-C. A previous study has described the expression of angiogenesis related factors (thymidine phosphorylase and bFGF) in stromal cells (macrophages, lymphocytes, and fibroblasts) in pancreatic cancer. ${ }^{22}$ In that study no correlation was seen between the expression of growth factors in the stromal cells and the IMD; however, thymidine phosphorylase expression in the stromal cells was found to be an independent predictor of survival. In addition, Tang and et al described a "faint VEGF-C immunoreactivity
... in some of the monocytes within the connective tissue around the cancer cells" in pancreatic cancer, ${ }^{23}$ but they did not further analyse this finding.

"Not only the cancer cells, but also the inflammatory cells, which express proangiogenic growth factors and accumulate in cases with higher IMD, influence the angiogenic properties of pancreatic cancer"

As reported previously, ${ }^{23-25}$ the results of our present study show that pancreatic cancer cells themselves are an important source of proangiogenic factors; in particular, we show a direct correlation between VEGF-A and bFGF expression in the cancer cells and the IMD, in accordance with previous studies. ${ }^{22} 26$ In addition, the results of our analysis suggest that not only the cancer cells, but also the inflammatory cells, which express proangiogenic growth factors and accumulate in cases with higher IMD, influence the angiogenic properties of pancreatic cancer. The evaluation of the expression of the above mentioned growth factors in the cancer cells revealed that the tumours analysed in our series exhibited a sort of angiogenic factor specific phenotype: that is, the number of VEGF-A positive inflammatory cells was higher in tumours with VEGF-A positive cancer cells and the number of bFGF positive inflammatory cells was higher in tumours with bFGF positive cancer cells. The chemotactic activity of VEGF-A, bFGF, and VEGF-C on inflammatory cells, which in turn express specific receptors for these factors, ${ }^{27} 28$ is well known. ${ }^{29}{ }^{30}$ The infiltration of inflammatory cells into the tumour tissue is often related to an increased expression of proangiogenic growth factors by the tumour cells. For example, a higher expression of VEGF-A by lung adenocarcinoma cells than by squamous cancer cells leads to a more abundant number of infiltrating mast cells, which in turn negatively affects the patient's survival. ${ }^{31}$ Another possible mechanism of inflammatory cell recruitment to tumour tissues is the expression of chemoattractant molecules by the tumour cells, as described for monocyte chemoattractant protein 1 in oesophageal cancer tissues and cell lines. ${ }^{32}$ Moreover, immune complexes made up of immunoglobulins and tumour associated antigens provide a further mechanism of inflammatory cell recruitment into the cancer tissue, and can directly promote the secretion of VEGF by macrophages. ${ }^{33}$

The capacity of mast cells and macrophages to produce proangiogenic growth factors has been described previously, ${ }^{34-36}$ and a correlation between the number of tumour infiltrating macrophages/mast cells and the IMD has been demonstrated in other human malignancies, including nonsmall cell lung cancer, ${ }^{31}{ }^{37}$ oesophageal cancer, ${ }^{14}{ }^{32}$ gastric cancer, $^{38}$ cervix and endometrial carcinoma, ${ }^{39}{ }^{40}$ and breast carcinoma. ${ }^{41}$ In addition, the mast cell specific proteases tryptase and chymase, both expressed by pancreatic mast cells, ${ }^{42}$ can promote angiogenesis ${ }^{43}{ }^{44}$; in particular, tryptase can specifically induce endothelial cell proliferation and capillary formation. ${ }^{45}$

The evaluation of the IMD has prognostic relevance in many human cancers, including pancreatic cancer. ${ }^{46-48}$ Moreover, the identification of tumours with high angiogenic activity could have therapeutic implications, particularly for tumours such as pancreatic cancer, where conventional cytotoxic treatments have limited efficacy. ${ }^{49}{ }^{50}$ Our results imply that the production of proangiogenic growth factors by the inflammatory cells could help define the angiogenic phenotype of a tumour, which could then be subjected to antiangiogenic treatment. In addition, the use of quantitative parameters, such as a count of the newly formed vessels (IMD) or of the number of infiltrating inflammatory cells, is 
more reproducible and could provide more useful information to the clinician than the semiquantitative assessment of proangiogenic factor expression by the tumour cells. In this series, the only factor that significantly affected the patients' survival was the presence of distant metastases. Interestingly, tumours with high numbers of infiltrating mast cells displayed a tendency towards a shorter survival than tumours with low numbers of infiltrating mast cells; moreover, tumours with high numbers of infiltrating mast cells/ macrophages and high IMD had a worse prognosis than tumours with low numbers of infiltrating mast cells/macrophages and low IMD. However, in both cases the differences in survival were not significant, probably because of the small number of cases analysed. These results suggest that inflammatory cells have a more detrimental than protective role in pancreatic cancer, as has already been shown in other human tumours. ${ }^{12-1431}$ Larger studies are needed to confirm the prognostic relevance of inflammatory cell infiltration in pancreatic cancer.

In our present study, mast cell numbers correlated with the presence of lymph node metastases, as has been described in squamous cell carcinoma of the oesophagus ${ }^{14}$ and lung adenocarcinoma. ${ }^{13}$ A possible explanation for this fact could be the ability of inflammatory cells to increase the number of peritumorous lymph vessels through the production of VEGF-C. Tumour associated macrophages have been found to express VEGF-C and its receptor (VEGFR3) and to have a direct influence on the process of lymphangiogenesis and lymphatic metastasis ${ }^{51}$; according to the results of this study, VEGF-C is also expressed by mast cells. The capacity to express VEGF-C seems to be acquired by the macrophages once they become part of the peritumorous inflammatory infiltrate and are under the influence of the tumour microenvironment, because their blood precursors are VEGF-C negative. ${ }^{51}$ However, we have found proangiogenic growth factor expressing inflammatory cells in the normal pancreas also. Stimuli related to normal tissue remodelling and renewal probably induce modification in the local population of inflammatory cells (for example, they can induce the expression of growth factors or enzymes that modify the interactions between the epithelial cells and the extracellular matrix), a process that will be amplified in the case of major tissue destruction, as happens in the vicinity of a developing tumour.

"Our results suggest that inflammatory cells have a more detrimental than protective role in pancreatic cancer, as has already been shown in other human tumours"

VEGF-C has been shown to stimulate the growth of intratumorous lymph vessels in a nude mouse model of breast cancer. ${ }^{52}$ Moreover, VEGF-C overexpression correlates with the rates of lymph node metastases in many human cancers, including pancreatic cancer. $^{23}$ Surprisingly, no correlation between VEGF-C expression by the tumour cells and the presence of lymph node metastases was found in our study. Instead, the number of VEGF-C positive inflammatory cells was higher in $\mathrm{N}+$ cases, a fact that underscores again the important role of the inflammatory infiltrate in influencing the biological properties of pancreatic adenocarcinoma.

In conclusion, we have shown an increase in the numbers of macrophages and mast cells in the stroma of pancreatic cancer in comparison with the normal pancreas. This accumulation seems not to be related to a concomitant condition of chronic inflammation of the pancreatic parenchyma, and could result from chemotactic stimuli released by the cancer cells. The inflammatory cells, through the expression of angiogenic growth factors, probably contribute
Take home messages

- There were significantly more mast cells and macrophages in pancreatic cancers than in normal pancreas and the number of mast cells correlated directly with the presence of lymph node metastases

- This accumulation was not related to chronic inflammation of the pancreatic parenchyma, and might result from chemotactic stimuli released by the cancer cells

- These inflammatory cells may influence the metastatic capacity of the cancer cells, and may contribute to the development of tumours with high angiogenic activity

- These tumours may benefit from antiangiogenic therapeutic strategies

to the development of tumours with high angiogenic activity, which may benefit from alternative therapeutic strategies.

\section{ACKNOWLEDGEMENTS}

This work was supported by grant number RF 01.140, Controllo qualità laboratorio oncologico: concerted action nazionale, definizione, interventi prioritari, metodologie e realizzazione; and grant number CTB 02.00336 ST 97, Italian National Research Council (CNR), Diagnostica molecolare in oncologia: sviluppo, validazione e applicazione della diagnostica molecolare in Oncologia.

\section{Authors' affiliations}

I Esposito, F Bergmann, Institute of Pathology, University of Heidelberg, 69120 Heidelberg, Germany

M Menicagli, N Funel, G Bevilacqua, D Campani, Department of Oncology, Transplantation and Advanced Technologies in Medicine, Division of Surgical, Molecular and Ultrastructural Pathology, University of Pisa, Pisa 56100, Italy

F Mosca, U Boggi, Department of Oncology, Transplantation and Advanced Technologies in Medicine, Division of General and Transplantation Surgery, University of Pisa

\section{REFERENCES}

1 Solcia E, Capella G, Klöppel G. Tumors of the exocrine pancreas. In: Rosai J, Sobin L, eds. Atlas of tumor pathology. Tumors of the pancreas. Washington DC: Armed Forces Institute of Pathology, 1997:64-88.

2 Ryu B, Jones J, Hollingsworth MA, et al. Invasion-specific genes in malignancy: serial analysis of gene expression comparisons of primary and passaged cancers. Cancer Res $2001 ; 61: 1833-8$.

3 lacobuzio-Donahue CA, Ryu B, Hruban RH, et al. Exploring the host desmoplastic response to pancreatic carcinoma: gene expression of stromal and neoplastic cells at the site of primary invasion. Am J Pathol 2002;160:91-9.

4 Gouon-Evans V, Lin EY, Pollard JW. Requirement of macrophages and eosinophils and their cytokines/chemokines for mammary gland development. Breast Cancer Res 2002;4:155-64.

5 Wong YC, Wang YZ. Growth factors and epithelial-stromal interactions in prostate cancer development. Int Rev Cytol 2000;199:65-116.

6 Farrow B, Evers BM. Inflammation and the development of pancreatic cancer. Surg Oncol 2002; 10:153-69.

7 Esposito I, Friess $\mathrm{H}$, Buchler MW. Molecular mechanisms in chronic pancreatitis. Zentralb/ Chir 2001;126:867-72.

8 Balaz P, Friess H, Buchler MW. Growth factors in pancreatic health and disease. Pancreatology 2001;1:343-55

9 Kleeff J, Kusama T, Rossi DL, et al. Detection and localization of Mip-3alpha/ LARC/Exodus, a macrophage proinflammatory chemokine, and its CCR6 receptor in human pancreatic cancer. Int J Cancer 1999:81:650-7.

10 Lin EY, Nguyen AV, Russell RG, et al. Colony-stimulating factor 1 promotes progression of mammary tumors to malignancy. J Exp Med $2001 ; 193: 727-40$

11 Westphal E. Über Mastzellen. Histologie und Klinik des Plutes: gesammelte Mitt(h)eilungen. In: Ehrlich P, ed. Farbenanalytische Untersuchungen. Zur Berlin: Hirschwald Press, 1891:17-21

12 Ribatti D, Ennas MG, Vacca A, et al. Tumor vascularity and tryptase-positive mast cells correlate with a poor prognosis in melanoma. Eur $J$ Clin Invest 2003:33:420-5.

13 Takanami I, Takeuchi K, Naruke M. Mast cell density is associated with angiogenesis and poor prognosis in pulmonary adenocarcinoma. Cancer 2000;88:2686-92. 
14 Elpek GO, Gelen T, Aksoy NH, et al. The prognostic relevance of angiogenesis and mast cells in squamous cell carcinoma of the oesophagus. $J$ Clin Pathol 2001;54:940-4

15 Esposito I, Kleeff J, Bischoff SC, et al. The stem cell factor-c-kit system and mast cells in human pancreatic cancer. Lab Invest 2002;82:1481-92.

16 Klöppel G, Hruban R, Adler G, et al. Tumours of the exocrine pancreas. In: Hamilton S, Aaltonen L, eds. World Health Organisation classification of tumours. Pathology and genetics of tumours of the digestive system. Lyon: IARC Press, 2000:220-30.

17 Sobin L, Wittekind C. TNM classification of malignant fumours, 6th ed. New York: Wiley-Liss, Inc, 2002.

18 Ochsenbein AF. Principles of tumor immunosurveillance and implications for immunotherapy. Cancer Gene Ther 2002;9:1043-55.

19 Dunn GP, Bruce AT, Ikeda $\mathrm{H}$, et al. Cancer immunoediting: from immunosurveillance to tumor escape. Nat Immunol 2002;3:991-8.

20 Ribatti D, Vacca A, Nico B, et al. The role of mast cells in tumour angiogenesis. Br J Haematol 2001;115:514-21.

21 Bingle L, Brown NJ, Lewis CE. The role of tumour-associated macrophages in tumour progression: implications for new anticancer therapies. J Pathol 2002; 196:254-65.

22 Fujioka S, Yoshida K, Yanagisawa S, et al. Angiogenesis in pancreatic carcinoma: thymidine phosphorylase expression in stromal cells and intratumoral microvessel density as independent predictors of overall and relapse-free survival. Cancer 2001;92:1788-97.

23 Tang RF, Itakura J, Aikawa T, et al. Overexpression of lymphangiogenic growth factor VEGF-C in human pancreatic cancer. Pancreas $2001 ; 22: 285-92$.

24 Itakura J, Ishiwata T, Friess $\mathrm{H}$, et al. Enhanced expression of vascular endothelial growth factor in human pancreatic cancer correlates with local disease progression. Clin Cancer Res 1997;3:1309-16.

25 Yamanaka $Y$, Friess $H$, Buchler $M$, et al. Overexpression of acidic and basic fibroblast growth factors in human pancreatic cancer correlates with advanced tumor stage. Cancer Res 1993;53:5289-96.

26 Fujimoto K, Hosotani R, Wada M, et al. Expression of two angiogenic factors, vascular endothelial growth factor and platelet-derived endothelial cell growth factor in human pancreatic cancer, and its relationship to angiogenesis. Eur J Cancer 1998;34:1439-47.

27 Jones $M$, Tussey L, Athanasou N, et al. Heparan sulfate proteoglycan isoforms of the CD44 hyaluronan receptor induced in human inflammatory macrophages can function as paracrine regulators of fibroblast growth factor action. J Biol Chem 2000;275:7964-74.

28 Akimoto S, Ishikawa O, lijima C, et al. Expression of basic fibroblast growth factor and its receptor by fibroblasts, macrophages and mast cells in hypertrophic scar. Eur J Dermatol 1999;9:357-62.

29 Gruber BL, Marchese MJ, Kew R. Angiogenic factors stimulate mast-cell migration. Blood 1995;86:2488-93

30 Skobe $M$, Hamberg $L M$, Hawighorst $T$, et al. Concurrent induction of lymphangiogenesis, angiogenesis, and macrophage recruitment by vascular endothelial growth factor-C in melanoma. Am J Pathol 2001;159:893-903.

31 Imada A, Shijubo N, Kojima H, et al. Mast cells correlate with angiogenesis and poor outcome in stage I lung adenocarcinoma. Eur Respir J 2000;15:1087-93.

32 Ohta M, Kitadai Y, Tanaka S, et al. Monocyte chemoattractant protein-1 expression correlates with macrophage infiltration and tumor vascularity in human esophageal squamous cell carcinomas. Int J Cancer 2002;102:220-4

33 Barbera-Guillem E, Nyhus JK, Wolford CC, et al. Vascular endothelial growth factor secretion by tumor-infiltrating macrophages essentially supports tumor angiogenesis, and $\lg G$ immune complexes potentiate the process. Cancer Res 2002;62:7042-9.

34 Moller A, Lippert U, Lessmann D, et al. Human mast cells produce IL-8. J Immunol 1993;151:3261-6.

35 Grutzkau A, Kruger-Krasagakes S, Baumeister H, et al. Synthesis, storage, and release of vascular endothelial growth factor/vascular permeability factor (VEGF/VPF) by human mast cells: implications for the biological significance of VEGF206. Mol Biol Cell 1998;9:875-84.

36 Leibovich SJ, Polverini PJ, Shepard HM, et al. Macrophage-induced angiogenesis is mediated by tumour necrosis factor-alpha. Nature 1987;329:630-2.

37 Chen JJ, Yao PL, Yuan A, et al. Up-regulation of tumor interleukin-8 expression by infiltrating macrophages: its correlation with tumor angiogenesis and patient survival in non-small cell lung cancer. Clin Cancer Res 2003;9:729-37.

38 Yano $\mathrm{H}$, Kinuta $M$, Tateishi $\mathrm{H}$, et al. Mast cell infiltration around gastric cancer cells correlates with tumor angiogenesis and metastasis. Gastric Cancer 1999;2:26-32.

39 Benitez-Bribiesca L, Wong A, Utrera D, et al. The role of mast cell tryptase in neoangiogenesis of premalignant and malignant lesions of the uterine cervix. $J$ Histochem Cytochem 2001;49:1061-2.

40 Tanaka Y, Kobayashi H, Suzuki M, et al. Thymidine phosphorylase expression in tumor-infiltrating macrophages may be correlated with poor prognosis in uterine endometrial cancer. Hum Pathol 2002;33:1105-13.

41 Valkovic T, Dobrila F, Melato $M$, et al. Correlation between vascular endothelial growth factor, angiogenesis, and tumor-associated macrophages in invasive ductal breast carcinoma. Virchows Arch 2002;440:583-8.

42 Esposito I, Friess H, Kappeler A, et al. Mast cell distribution and activation in chronic pancreatitis. Hum Pathol 2001;32:1174-83.

43 Taipale J, Lohi J, Saarinen J, et al. Human mast cell chymase and leukocyte elastase release latent transforming growth factor-beta 1 from the extracellular matrix of cultured human epithelial and endothelial cells. J Biol Chem 1995;270:4689-96.

44 Muramatsu M, Katada J, Hattori M, et al. Chymase mediates mast cellinduced angiogenesis in hamster sponge granulomas. Eur J Pharmacol 2000;402:181-91.

45 Blair RJ, Meng H, Marchese MJ, et al. Human mast cells stimulate vascular tube formation. Tryptase is a novel, potent angiogenic factor. J Clin Invest 1997; $99: 2691-700$

46 Ikeda N, Adachi $M$, Taki T, et al. Prognostic significance of angiogenesis in human pancreatic cancer. Br J Cancer 1999;79:1553-63.

47 Linder S, Blasjo M, von Rosen A, et al. Pattern of distribution and prognostic value of angiogenesis in pancreatic duct carcinoma: a semiquantitative immunohistochemical study of 45 patients. Pancreas $2001 ; 22: 240-7$.

48 Khan AW, Dhillon AP, Hutchins R, et al. Prognostic significance of intratumoural microvessel density (IMD) in resected pancreatic and ampullary cancers to standard histopathological variables and survival. Eur J Surg Oncol 2002;28:637-44.

49 McKenna S, Eatock $M$. The medical management of pancreatic cancer: a review. Oncologist 2003;8:149-60.

50 Rosenberg L, Lipsett $M$. Biotherapeutic approaches to pancreatic cancer. Expert Opin Biol Ther 2003;3:319-37.

51 Schoppmann SF, Birner P, Stockl J, et al. Tumor-associated macrophages express lymphatic endothelial growth factors and are related to peritumoral lymphangiogenesis. Am J Pathol 2002;161:947-56.

52 Skobe M, Hawighorst T, Jackson DG, et al. Induction of tumor lymphangiogenesis by VEGF-C promotes breast cancer metastasis. Nat Med 2001; 7:192-8 